\title{
Optical Monitoring System for Scalable All-Optical Networks
}

\author{
Abdelhafid Amrani, Jesús Roldán, Member, IEEE, and Gabriel Junyent
}

\section{Introduction :}

High traffic and high capacity communications are believed to become cost-effective with the use of all-optical networks based on OFDM (Optical Frequency Division Multiplexing). In such networks, new strategies for OA\&M (Operation, Administration and Maintenance) functions need to be developed so as to suit the introduction of a new optical layer where in wavelength routing is exploited and faults are detected in a transparent manner [1].

In this paper, we propose a scheme to monitor optical channels in the optical layer for supporting OA\&M functions of a transparent and scalable photonic network.

\section{Description of the optical monitoring system (OMS) :}

In all-optical networks, a link by link optical monitoring system will be of great usefulness to detect degradations through the network and facilitate the identification of faulty elements so that an autonomous fault restoration could be performed in the optical layer and without having recourse to an electronic layer (SONET/SDH or ATM) at which bottleneck may occur when electrical interfaces process high data traffic.

The OMS proposed in this letter, is distributed along the optical path crossconnects with add-drop capability. Each of these local nodes is responsible for monitoring a part of the spectrum utilized by the network. A centralized supervisor system receiving information from the local monitoring systems (by a service-bearing channel), can display the spectrum of all network transmitters. The monitoring operation is simultaneous in sense of independent local monitoring systems, and is performed as follows, Fig(1) : Every local node contains a frequency sweeping laser which scans with a ramp waveform a spectrum range to which belongs a set of specified wavelengths, for example the local transmitter's spectrum. By heterodyning the frequency scanning laser with the local carriers and low pass filtering the resulting signal, pulses occur in the time domain when coincidence is held between the heterodyned frequencies. Therefore, the shape of the detected pulse indicates channel presence or loss, thus can inform about a link or transmitter fault. Moreover, channel power degradation could also be pointed out by comparing the detected optical power in the monitored spectrum to a noise floor determined by the power detected in a non-occupied channel. In this way, a SNR (signal to noise ratio) estimation is provided alloptically by the proposed OMS.

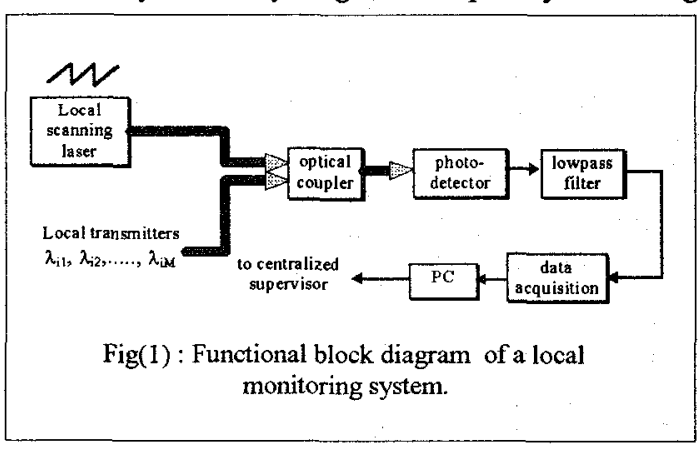

\section{Experimental result :}

In this section, we aim at demonstrating experimentally the feasibility of the proposed OMS in a local node. For the others nodes, it will be the same except for the monitored wavelength range which will be chosen to fit another part of the spectrum utilized by the optical network.

The transmitter and frequency scanning lasers are tunable DFB lasers from GEC-Marconi. The frequency scanning range is adjusted to $12,5 \mathrm{GHz}$ because of monitoring only one local transmitter, although it could be enlarged if needed. The period to sweep the frequency scanning range is set at $150 \mathrm{~ms}$. Data acquisition and channel supervision are implemented with a PC card and a windows environment software. Fig (2) shows the experimental result of the local monitored spectrum of a pseudorandom FSK

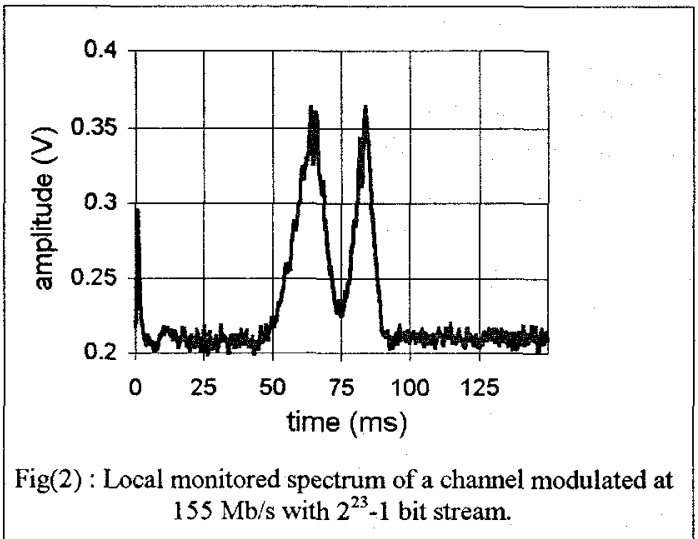
wide deviation modulated channel at $155 \mathrm{Mb} / \mathrm{s}$ with $2^{23}-1$ bit stream. Proper operation of this OMS was also verified experimentally for $622 \mathrm{Mb} / \mathrm{s}$ and $2.5 \mathrm{~Gb} / \mathrm{s}$ SONET/SDH transmission velocities. 
The present OMS exploits spectrum partitioning by distributing monitoring function among the local nodes. Therefore, the total monitored spectrum could be enlarged as the number of nodes is elevated allowing scalability and modularity of the OMS. Another advantage of a distributed monitoring system is its faster response time in comparison to a centralized monitoring system.

\section{Link fault detection :}

A wavelength channel carrying traffic between two nodes, if not monitored in the local OMS of the node destination informs that the link joining the two nodes is failed.

Let us see how link fault can be automatically detected by the present method in the case of an optical ring network. The ring architecture is attractive for its failure restoration capability and for using a minimized number of links. Fig (3) shows a self healing bi-directional OFDM optical ring network with its wavelength matrix for a full mesh traffic pattern.

Among several possibilities, a link by link fault detection can be accomplished as shown in Fig(4). Note that only two local optical monitoring systems (of nodes 1 and 3 in the example) are sufficient to inform about the health of all links in the network, when they monitor some specified channels received in their corresponding nodes (a link fault is assumed to be a simultaneous fault in the two fibers forming the link).

This method can be generalized to an arbitrary topology network by corresponding to each link a particular wavelength (used also for traffic transmission) and a particular local OMS.

Another advantage of the present scheme is its ability to form part of a frequency control and measurement system which is important for reducing costs of network OA\&M system: Indeed, by heterodyning separately the sweeping laser (in Fig(1)) with a reference frequency, the spectral positions of the monitored carriers can be compared with the reference frequency, thus can be accurately measured and locked to precise off-set values from the reference frequency [2][3].

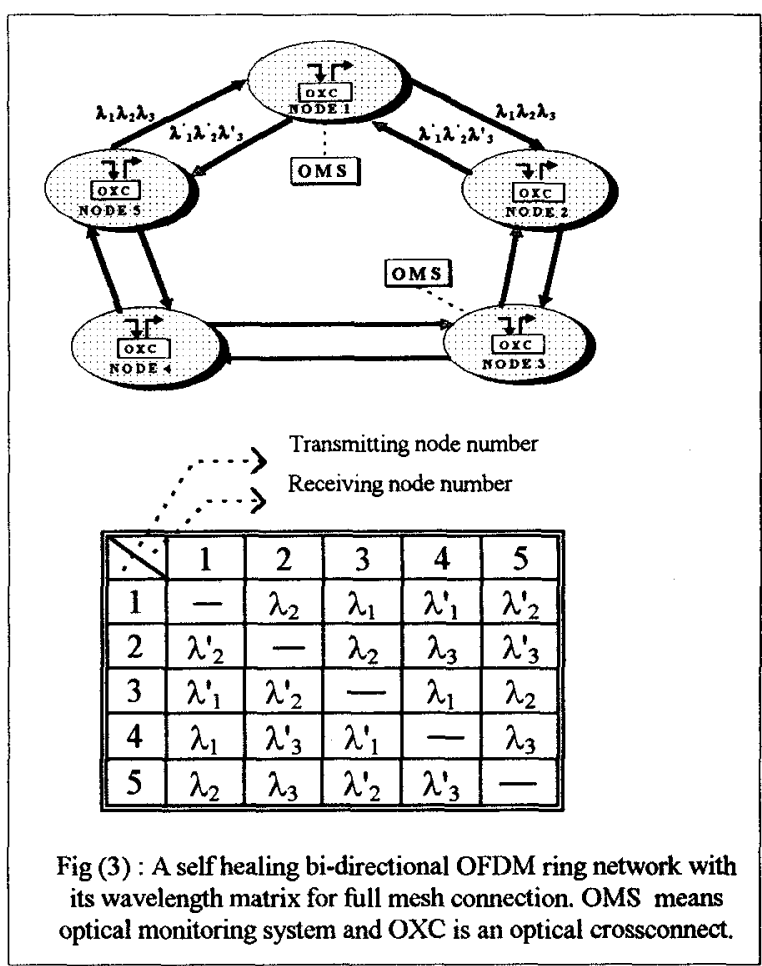

\begin{tabular}{|c|c|c|}
\hline $\begin{array}{c}\text { LOCAL OMS OF } \\
\text { NODE }\end{array}$ & $\begin{array}{c}\text { NO CHANNEL } \\
\text { DETECTED }\end{array}$ & $\begin{array}{c}\text { ALARM: } \\
\text { LINK FAIIED }\end{array}$ \\
\hline 1 & $\lambda_{2}^{\prime}, \lambda_{1}^{\prime}$ & $1-2$ \\
\hline 1 & $\lambda_{1}^{\prime}$ & $2-3$ \\
\hline 3 & $\lambda_{1}^{\prime}$ & $3-4$ \\
\hline 1 & $\lambda_{1}$ & $4-5$ \\
\hline 1 & $\lambda_{2}, \lambda_{1}$ & $5-1$ \\
\hline
\end{tabular}

Fig(4) : link by link failure detection $i-j$ means the link joining nodes $i$ and $j$.

\section{Conclusion :}

We have proposed an optical monitoring system (OMS) for scalable all-optical networks and experimentally demonstrate its feasibility in a local node. We have also described its application in a self healing optical ring network.

The proposed OMS presents interesting advantages like scalability and modularity, unlimited total monitored spectrum range, suitability for dense frequency spaced channels, and link or transmitter fault detection.

\section{References:}

[1] Y. Tada et al. OA\&M Framework for Multiwavelength Photonic Transport Networks. IEEE Journal on Selected Areas in Communications, pp. 914-921, Vol.14, No.5, June 1996.

[2] A. Amrani et al. Multifrequency Absolute Stabilization for Photonic Communication Networks. Proceedings of IEEE International Conference on Communication Systems, pp 949-951, Singapore, November 1996.

[3] A. Amrani et al. Accurate Frequency Measurement for Photonic Network Management. Proceedings of the International Conference of Fiber Optics \& Photonics. Vol 1, pp 552-555. Madras, India, December 1996. 